Christian Fischer · Karl-Peter Fritz - Peter Eberhard • Heinz Kück

\title{
Investigation and Design of an Impact Actuated Micro Shift Valve
}

Received: 23 December 2012 / Accepted: 27 February 2013 / Published: March 2013

\begin{abstract}
New concepts for an impact actuated micro shift valve are presented which are useful e.g. as implant for the hydrocephalus disease. Such an implant must fulfil requirements, such as using biocompatible materials, a separation of the actuator from the fluid, MRT safety and low energy consumption in order to allow a battery powered system. The concepts are based on impacts that transmit an impulse into the interior of the valve through the casing, switching the valve. In order to predict the energy transmitted into the valve, an elastic multibody model is created, verified with full finite element simulations and experiments on scaled-up models. Using this model, the most important effects and parameters are discussed. Also, fluid effects are included into the elastic multibody model for a qualitative assessment of its influence on the efficiency. The simulations are compared to experiments performed with a scaled model for two different cases. Two designs of a shift valve based on impact actuation are built as prototypes and tested.
\end{abstract}

Keywords micro shift valve - impact actuation · multibody dynamics · squeeze-film effect · experiments

C. Fischer, P. Eberhard

Institute of Engineering and Computational Mechanics, University of Stuttgart, Pfaffenwaldring 9, 70569 Stuttgart

URL: http://www.itm.uni-stuttgart.de

E-mail: christian.fischer@itm.uni-stuttgart.de

E-mail: peter.eberhard@itm.uni-stuttgart.de

K.-P. Fritz, H. Kück

Institute for Micro Assembly Technology, Hahn Schickard Gesellschaft, Allmandring 9B, 70569 Stuttgart

URL: http://www.hsg-imat.de

E-mail: fritz@hsg-imat.de

E-mail: kueck@hsg-imat.de

\section{Introduction}

In many technical devices or systems shift valves are used to control the fluid flow. Due to the multiple applications, they can be found in many areas of industry and everyday life, such as air conditioning systems, drinking water systems, cooling circuits of machines or household appliances. One important aspect for some applications of shift valves is media separation, meaning that there is no contact between the environment and the fluid within the valve. This is important, e.g. in cases where purity demands for the media are extreme or the fluid is harmful. State-of-the-art shift valves with media separation usually contain deformable membranes which either block or enable the flow. Hereby the actuation of the membrane often is done by a solenoid. When using a solenoid as an actuator for a membrane valve one faces the disadvantage that the membrane keeps the deformed state only if energy is supplied to the system.

A very special application for shift valves is the therapy of the hydrocephalus disease. Hereby, an implanted shift valve is used as a shunt that drains off the excessive cerebrospinal fluid from the ventricular system of the patient. Today passive shunt valves are available whose opening characteristics are controlled by the static pressure in the ventricular system and, therefore, cannot be shifted from outside. Yet this option to actively shift the valve should open new therapy and diagnosis options for the hydrocephalus disease. Additional to the above mentioned media separation in this application, the MRT-safety of the valve is required, meaning that no ferromagnetic material is allowed in the shift valve, as this would execute a harmful force on the tissue when exposed to the strong magnetic fields in an MRT apparatus.

A new concept is presented in the following based on impact actuation, especially meeting the requirements of an implantable, actively shiftable valve. However, it can also be used in other applications. The successful design of such a valve depends on a deeper understand- 
ing of the parameters and effects occurring during impact. Therefore an elastic multibody model is created capable of capturing the most important effects. This model is validated by experiments and verified by full finite element simulations on a scaled-up model. It is also extended to include fluid effects such as the squeeze-film effect. The gained understanding of impacts is used to create two designs which are not only simulated, but also tested with demonstrators.

\section{Concepts for an impact actuated shift valve}

During the investigations made to understand the shifting process and the impact, different valve designs were made. The principle of the valve and the boundary conditions for the development are listed at first. Different impact actuators that might be used are explained briefly. Some of the analysed designs are introduced here which hopefully makes it easier to understand the following simulation models and experiments.

\subsection{Principle of operation}

The proposed concept of a shift valve with separated media is based on a body inside the valve which can be in two distinct positions. One allowing fluid flow and one blocking the fluid flow as depicted in Fig. 1. It is the basic idea to transmit energy by an impulse wave or deformation from actuators ('open' and 'close') into the valve chamber to the sphere which will switch its position. A spring in the valve chamber prevents the sphere from an unintended shifting. On the other hand, the spring must not be too stiff, so that shifting is not impaired.

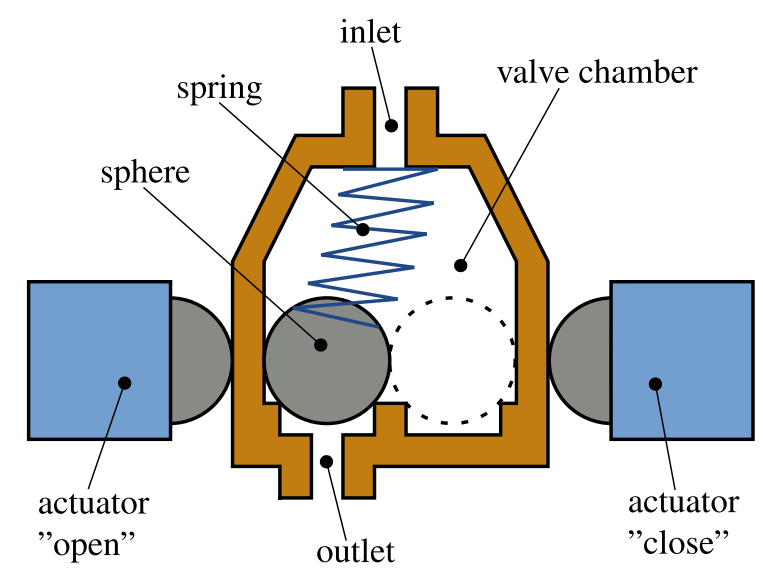

Fig. 1 Concept of the shift valve

Besides showing media separation, this valve concept has the following advantages:
- All parts of the valve that come into contact with the fluid can be made of inert materials such as stainless steel, titanium or plastic material that do not show harmful interactions with the fluid. In particular the materials can be chosen with respect to the requirement of the above mentioned MRT safety.

- The characteristics of the impact actuation allows the overcoming of breakaway effects of the sphere as it provides the biggest force on the sphere at the beginning of its movement.

- As for the bistable shifting the actuation units need to be provided with energy only to initiate the shifting. After being shifted the valve doesn't consume energy which leads to a very energy efficient behaviour especially for applications where the state of the valve is to be maintained for a long time.

- Nearly all types of impact actuators can be used to initiate the shifting of the valve. Piezoelectic actuators are possible as well as hydraulic, electromagnetic, pneumatic or even manually powered actuators. By this a very modular set-up of the valve is reached which even gives the possibility to establish the valve chamber as a disposable part which is reversibly placed between two impact actuators and is discarded after a certain period of usage.

\subsection{Impact actuators}

With respect to the requirement of MRT safety for an implantable device, two different piezoelectric actuators were investigated as they contain no ferromagnetic materials. Besides this, an electromagnetic impact actuator with a lift magnet was investigated as a possible actuator for many other applications. This type of actuator is suitable for most industrial applications, as it is available at relatively low cost and also has no special requirements concerning the electronics.

As usual in active implants, the required high voltage for the piezoelectric actuators (about 150 Volts) has to be generated from a battery. This leads to an electric circuit with a step-up converter, which however, has big differences for the two different types of piezo actuators.

\subsubsection{Piezo stack actuator}

The impact with a piezo stack actuator is performed by abruptly applying a high voltage to the piezoceramics that leads to a very fast elongation of the piezo stack. The biggest advantage of this type of actuator is the high potential for miniaturisation and a high energy density. Yet, because of the very small strokes that are in the micrometer range the adaptation to a valve setup is very critical, as no geometrical tolerances from the fabrication and assembly process can be compensated. Furthermore, the electrical control of the actuator has to provide the driving voltage of the piezo 
stack actuator before applying it which requires a welldimensioned buffer capacitor within the electrical control. A schematic view of the control circuit is shown in Fig. 2

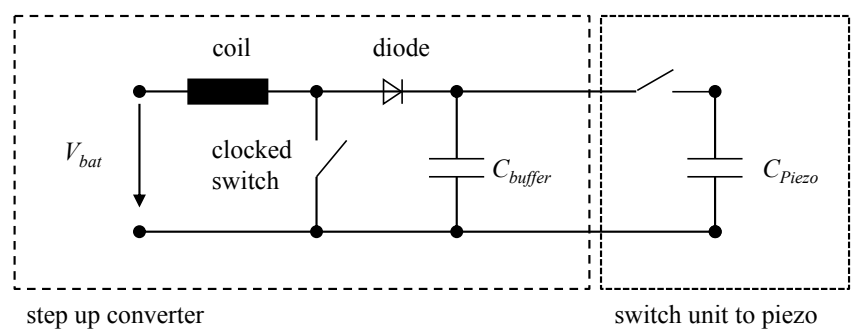

Fig. 2 Schematic view of the control circuit for a piezo stack actuator

\subsubsection{Piezo bending actuator}

Compared to a piezo stack actuator, the piezo bending actuator can be driven with a much simpler electrical circuit as it can directly be used as the high-voltage capacitor in the step-up circuit that transforms the supply voltage of the battery. During the high-voltage generation, the piezo bending actuator increasingly deflects until the required high voltage is reached. Then, the stored electric charge is discharged via a resistor with a time constant that is shorter than the mechanical time constant of the deflected bending actuator. This allows the piezo bending actuator to swing back unimpeded. When reaching the straight position, the bending actuator has its highest velocity and performs an impact to the wall of the valve chamber. As the velocity does not decrease too much near the straight position, this setup is able to compensate assembly tolerance in a quite wide range. A schematic view of the control circuit for the piezo bending actuator is shown in Fig. 3 .

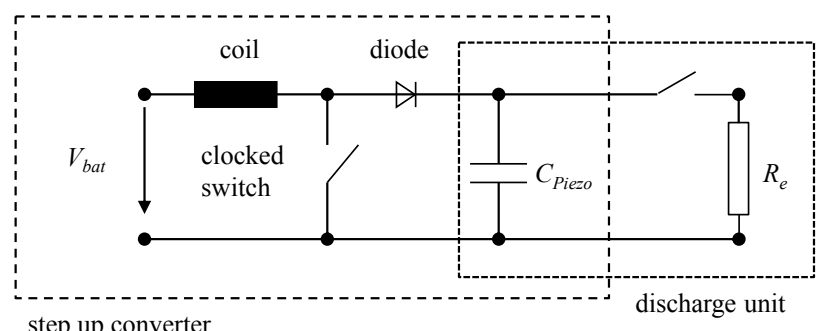

Fig. 3 Schematic view of the control circuit for a piezo bending actuator

Compared to the piezo stack actuator, the piezo bending actuator is the preferred alternative for an implantable shift valve with impact actuation.

\subsection{Design of the valve chamber}

As can be seen in Fig. 11 the sphere in the valve is in a statically overdetermined position when closing the outlet of the valve. According to this, it is hardly possible that the sphere is in proper contact to the wall of the valve chamber where the impact transmission takes place and at the same time perfectly seals the outlet of the valve because of the geometrical tolerances of the components of the valve.

To overcome this problem, a detailed analysis of the valve design was performed by Fritz 4 . The basic idea of this analysis was to define different subfunctions of the valve, to find possible solutions for each subfunction and to combine selected solutions to an overall design concept. The results were partially used also in Fritz and Fischer [5]. Two designs are chosen and presented here.

\subsubsection{Valve design with a rocker element}

Figure 4 shows a cross-sectional view of a shift valve with a rocker element. In this design, the subfunctions 'sealing of the valve outlet' and 'transmission of the impact energy' are distributed to two functional parts within the valve chamber. The impact energy is transmitted to the sphere which again is fixed in two distinct positions by a spring. When shifting the position, the sphere tilts the rocker element and thereby opens or closes the fluidic path through the valve chamber. Possible geometrical tolerances only lead to a slight misalignment of the contact point of the sphere at the wall of the valve in vertical direction.

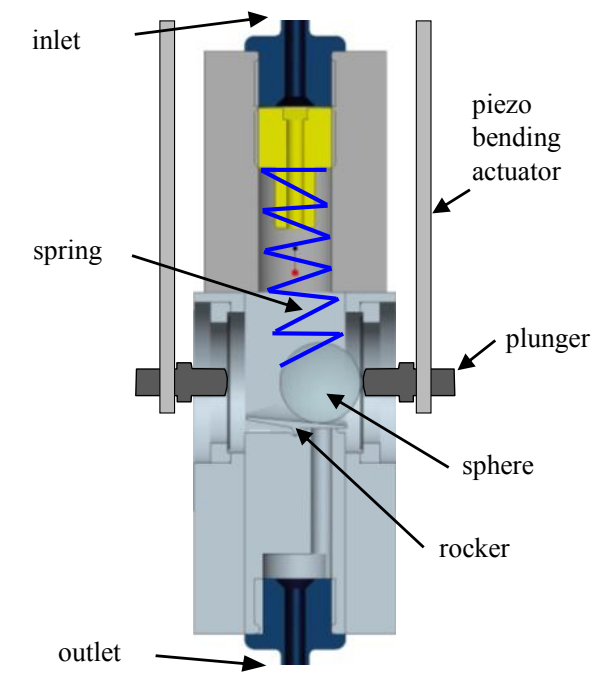

Fig. 4 Cross sectional view of the valve design with a rocker element

Besides the advantageous separation of functions, this valve design also has the following benefits: 
- The part of the valve chamber's wall, where the impact energy is transmissed can be designed and optimized widely independent from the rest of the valve. In particular it can be a thin membrane that is inserted into the valve body.

- The set-up of the whole valve can be realized in a modular way as shown in the exploded view of the valve in Fig. 5. Thereby it has to be considered that the overall dimensions of the shown model $(34 \times$ $17 \times 11.7 \mathrm{~mm}^{3}$ ) are much bigger than necessary. This is because the set-up was designed as a functional demonstrator where the different components of the valve and the actuation units can be changed easily and are connected with screws. Besides this demonstrator, a miniaturized set-up was investigated with overall dimensions of the valve chamber of only $4.2 \times$ $8 \times 8 \mathrm{~mm}^{3}$.

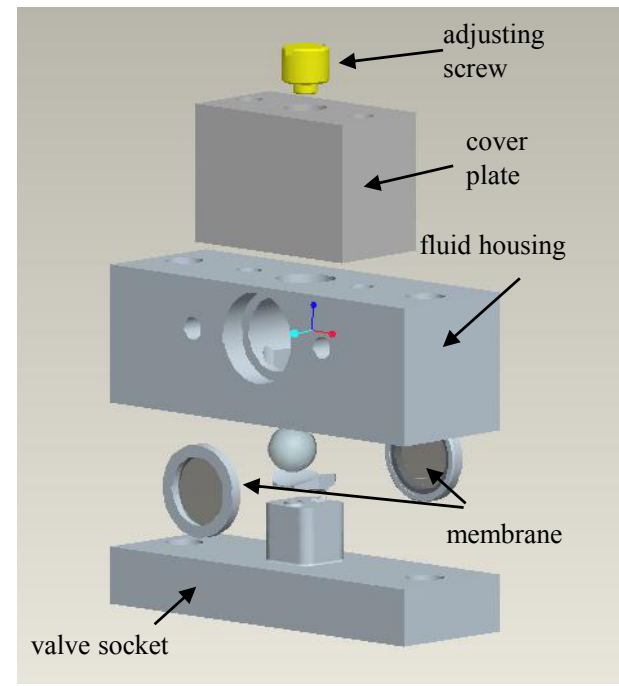

Fig. 5 Exploded view of the valve design with a rocker element

\subsubsection{Design with flexible mounted seal seat}

This concept is an advancement of shift valves with inserted seal seats into the valve body, as depicted in Fig. 6. Both seal seats are part of an island which is flexible connected by a membrane with the shift valve body. The advantages of this concept are better flushing due to smallest dead spots, the potential to work with only one actuator and its modular set-up, because of splitting the system into a reusable drive and a disposable shift element. This combined with injection moulding as a process of manufacture for the shift element promises a cost-effective system. The switching element (a sphere) is provided in one of both seats that are conical in shape with an angle of aperture $\gamma$ and an angle $\alpha$ compared to the horizontal line. The sphere is pushed into one of the seats by a spring and seals it. Below the island and at the outside of the valve is a lift magnet actuator. Both angles $\gamma$ and $\alpha$, together with the spring force and its pre-load, were optimised for a defined take-off speed of the sphere that can be controlled by the supply voltage of the lift magnet actuator. The impulse of the actuator results in a motion of the sphere with initial angle $\beta$ of the sphere based on the theory of slate-throwing.

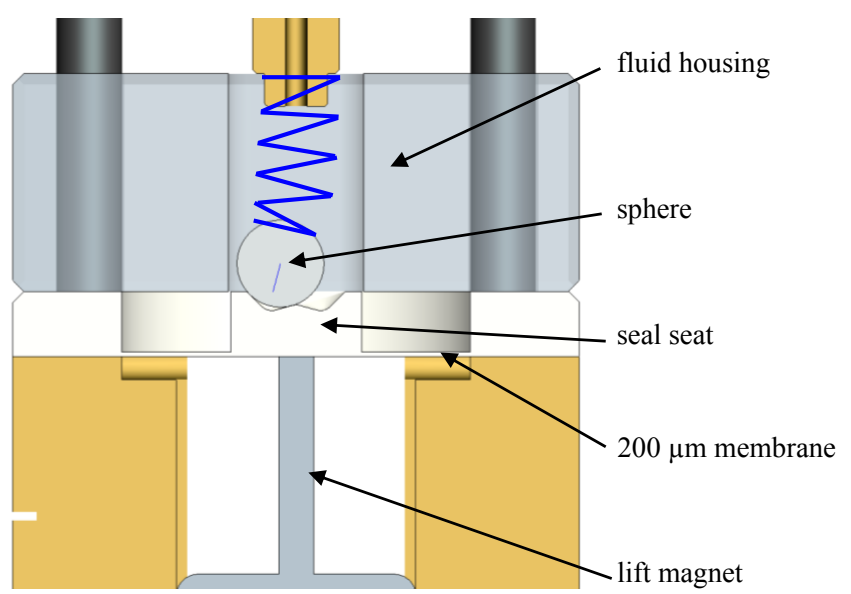

Fig. 6 Cross section of the shift valve design

\section{Multibody model for the impact and experimental verification}

The most important part in the simulation of this system is the impact and the impulse transmission through the casing of the valve. To achieve an understanding of the impact behaviour and the parameters influencing it, a flexible and fast model which also allows optimisations and parameter studies must be created. Usually, detailed analyses of impact are done with finite element simulations, allowing complex models of the geometry, contact and material properties. However, these models require long times in order to recreate reliable meshes each time the geometry is modified. Furthermore, the simulation of $2 \mathrm{D}$ or even $3 \mathrm{D}$ transient contact simulation takes a lot of computational time. This is why a simpler model is developed in this section. Elastic multibody systems allow us to reduce the model complexity and simulation time without sacrificing accuracy too much. These models were partially introduced by Eberhard and Fischer 2 .

In this work, finite element simulations are only used to validate the simplified models and whenever the geometry is more complex requiring a $3 \mathrm{D}$ FE simulation. The model used for the simulations is shown in Fig. 7. The impact, being the beginning of a shift process is modelled using two sphere-like bodies and a circular plate. 


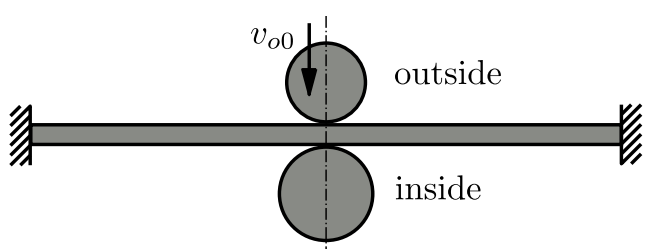

Fig. 7 Model for impact simulations

The outer body has the initial velocity $v_{o 0}$. The simplification of the actuator as a sphere with initial velocity is chosen in order to separate the impact process from additional effects of piezo stack actuators and piezo bending transducers. There are two contacts at the same time, one between the outer body and the plate and the other between the plate and the body inside the valve chamber. The most significant result of the simulation is the energy transmitted from the outer body to the inner body, defined as the efficiency

$$
\eta=\frac{T_{i 1}}{T_{o 0}}=\frac{m_{i} v_{i 1}^{2}}{m_{o} v_{o 0}^{2}} .
$$

This efficiency is the ratio of kinetic energy $T$ of the colliding bodies with mass $m$ and velocity $v$. The subscript denotes the state just before impact (0) and after impact (1). This ratio is very sensitive to the velocities, which means that the contact must be modelled in a detailed manner. It will be shown that the full 3D finite element model is not necessary to capture the relevant effects and a simpler model gives high accuracy whenever the bodies consist of the two spheres and a plate.

But first, the effects are discussed which can be expected in impact processes which are highly dynamic. The most important effect is the elastic deformation of the impacting bodies. We separate it into two different parts. First, the global deformations like vibrations or wave propagation within the bodies and second, the local deformations in the contact region. Typically, the local elastic deformations do not dissipate energy but vibration and wave propagation are the key to capture dissipation because this energy remains in the bodies after the impact. The local deformations are influenced by all sorts of effects which are not captured in standard linear elasticity which is also used in the Hertzian contact law, see Goldsmith [6] and Szabó [13].

Plastic deformations occur almost always due to the small area of impact and are specifically important if multiple plastic impacts occur at the same location. As a further complication, due to the high speed and high strain rate $\dot{\epsilon}$, nonlinear material effects like viscoplasticity must be considered too, if plasticity is modelled. See Minamoto et al [9] for a detailed analysis of these effects. Typically, the difference between elastic and plastic simulations are especially large for the first few impacts. After several impacts, less plastic deformations occur because the impact area gets larger and the stresses are therefore reduced.
Global effects like vibrations and wave transmission are very important for bodies where the periods of the eigenfrequencies are longer than the contact time. If waves are reflected back and forth multiple times during contact, the bodies are in a quasi-static state, see Goldsmith [6]. Especially, spheres fall in this category, which means that they can be modelled as rigid bodies. Elastic bodies can describe wave effects, if the model is detailed enough to capture the waves.

We will model the contact with an elastic plate using axisymmetric plate elements with different numbers of degrees of freedom and the Hertzian contact law. This model is set up and simulated using the numerical library SciPy [8] and the symbolical library SymPy [12]. The reference solution for the simulations is an explicit finite element simulation using Abaqus. An easier approach for modelling impact in multibody systems is the use of the coefficient of restitution which is based on assumptions about the expected impulse losses. Using this coefficient, the Hertzian contact law is often modified to incorporate these assumed losses. This does not seem to be useful here, because the losses are the result of the simulation and therefore unknown.

\subsection{Multibody model with elastic plate and Hertzian contact}

As already motivated, a simplified elastic multibody model is used primarily to model the contact of three colliding bodies. It consists of three bodies, an outer body, modelled as a point mass, or an elastic beam with attached point mass. The second body is a circular plate and inside the valve is another point mass. This model is sketched in Fig. 8

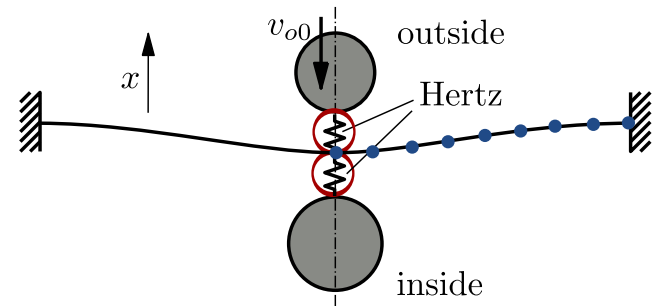

Fig. 8 Axisymmetric elastic multibody model with Hertzian contact and axisymmetric finite plate elements

The elastic plate is modelled with plate finite elements proposed by Zienkiewicz et al [14]. The mass matrix is implemented according to Hinton [7. The resulting linear system for the plate is transformed to modal coordinates. This gives the advantage of much faster time integration and the possibility to perform a modal reduction by transforming the model to a subspace of the modal coordinates. Basically, high frequency eigenmodes are assumed to be negligible. By doing that, the 
time integration is even faster and the influence of the number of eigenmodes can be studied. This is particularly important here because by doing that, the influence of different number of modes can be investigated. This approach of using a modal model for the global behaviour and a local contact force law such as the Hertzian law for the local behaviour is described in Seifried et al [11] and Seifried [10. However, the transformation to modal coordinates is not the only possibility. In Fehr and Eberhard [3] more powerful methods for transformation are presented. By using such methods, the number of ansatz functions can be decreased.

As replacement for the outer point mass, an elastic beam with attached point mass can be selected. Material damping can be considered for the plate by using modal damping. Additionally, in Section 3.5 a model for squeeze-film flow is introduced which can be activated if needed.

\subsection{Finite Element Model}

The reference solution is computed with the commercial finite element program Abaqus. The simulation is an explicit dynamic simulation, using a linear elements and a linear elastic material model. The axisymmetric models are set up with axisymmetric elements which simplifies meshing with quadrilaterals and allows high-quality meshes. Three-dimensional meshes are only used when necessary and the mesh is created with tetrahedral elements.

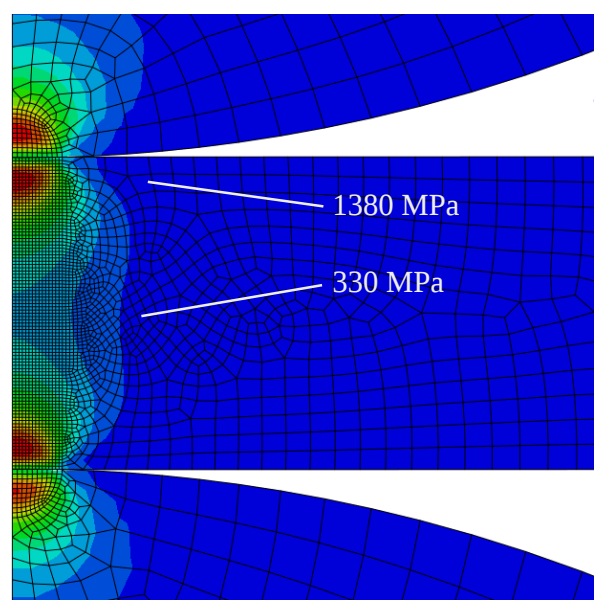

Fig. 9 Example of a mesh and the resulting stress distribution

The elements have linear geometry and linear ansatz functions. The contact is modelled using the kinematic master-slave contact algorithm provided by Abaqus. This contact algorithm prevents penetration by using a predict corrector method. The kinematic state of the model is advanced in the predictor phase ignoring contact. After that, the corrector part applies an acceleration to the master and slave nodes eliminating the penetration. This is very accurate compared to traditional penalty approaches, where the penalty factor must be chosen, and typically leads to very stiff systems with increased computational time. The mesh is refined in the contact region so that approximately 10 to 15 elements are across the contact radius which gives according to Seifried et al [11] and Seifried [10] sufficiently accurate results. A typical mesh with the stress distribution is shown in Fig. 9. In order to capture wave effects, the largest elements must be small enough to capture wave effects by discretising expected waves with at least 20 elements along the wavelength.

It is the primary goal of this model to verify the simpler elastic multibody model and simulate 3D models with complex geometry which cannot be simulated with the elastic multibody model.

3.3 Comparison of the multibody model and the finite element model

The multibody model is compared to the full transient finite element (FE) simulation. This allows to determine the accuracy of the multibody model compared to the full FE simulation, which has fewer simplifications. The simulations are later also compared to experiments, where it is better to use larger models which are easier to handle and give more accurate results. That is why the simulation models are also compared using this scaled-up model. To get a scaled-up model, the plate is scaled such that it has the same $R / t$ ratio. The spheres are scaled such that the resulting contact time has the same ratio to the period of the first eigenfrequency. The material for all bodies is steel with density $7780 \mathrm{~kg} / \mathrm{m}^{3}$, Young's modulus $210 \mathrm{GPa}$ and Poisson ratio 0.3. The material and geometrical data of the chosen model are summarized in Table 1 for two different sphere radii and their respective initial velocity.

Table 1 Geometrical data and initial condition of the scaled model

\begin{tabular}{lrrr}
\hline & outer sphere & plate & inner sphere \\
\hline$r[\mathrm{~mm}]$ & $6.35 / 10$ & 33 & $6.35 / 10$ \\
$t[\mathrm{~mm}]$ & - & 2 & - \\
$v_{0}[\mathrm{~mm} / \mathrm{s}]$ & $-437 /-550$ & 0 & 0 \\
\hline
\end{tabular}

Some simulation results are shown in Fig. 10 for the sphere with $10 \mathrm{~mm}$ radius. The three figures have the same time-scale and show the displacement, velocity and contact force for three different simulations for all three bodies. A reference simulation is performed by the finite element model and two multibody simulations with different degrees of freedom for the plate. Model A has only one degree of freedom using the first eigenfunction of the fixed circular plate. Model B uses 
the first 16 eigenfunctions of the plate which suffices to represent the behaviour of the finite plate elements with very little error. On the first glance, it is apparent that model B is in good agreement with the FEM and model A is obviously too simple. The resulting efficiencies as defined in Eq. (1) are displayed in the grey boxes. Again, it is clear that model B and the FEM are in good agreement but model A is not good enough. However, it already represents the very basic effects, showing that the first eigenfrequency plays a very important role. From now on, we will only refer to the multibody model with a sufficient number of eigenfunctions for the plate - model $\mathrm{B}$. The velocity curve of the FE simulation, compared to the multibody model, contains high-frequency oscillations which are not damped in the explicit FE solver.

A more detailed investigation shows that the multibody model has a slightly longer contact time and lower contact forces. These differences are caused by the stiffness of the plate in longitudinal (thickness) direction, being neglected in the plate elements used in the elastic multibody model. This influences the stress distribution of the Hertzian contact law which assumes an infinite half space. In Fig. 9, the problem can be seen clearly. The stress between the two contact points is not zero; therefore, the stress distributions influence each other. The assumption in the Hertzian law would require zero stress between the contact points.

The stresses occurring in the simulation models are well above yield, so that plastic deformations occur. Elastic, ideal-plastic behaviour is not precise enough due to the extremely high strain rate $\dot{\epsilon}$. Therefore, the material must be modelled visco-plastic. Further, multiple contacts occur which lead to an effect called shakedown which basically deforms the colliding bodies plastically during each impact until the geometry has changed enough, so that the stress is below yield and the impact is elastic again. Simulations with plastic material behaviour also show these effects. However, detailed material parameters for different strain rates are required to compare the results with experiments.

\subsection{Comparison with experiments}

In computational models, simplifications are necessary and useful to reduce the model complexity. However, the assumptions must be verified. So it is good practice to compare simulations to experiments wherever possible. An experimental set-up is used similar to the set-up used by Seifried et al [11] which is based on laser Doppler vibrometers (LDVs) to measure displacements and velocities at high frequencies $(f<100 \mathrm{kHz})$.

\subsubsection{Experimental set-up}

The set-up is shown in Fig. 11 with the plate and the release mechanism. The two LDVs are mounted to the
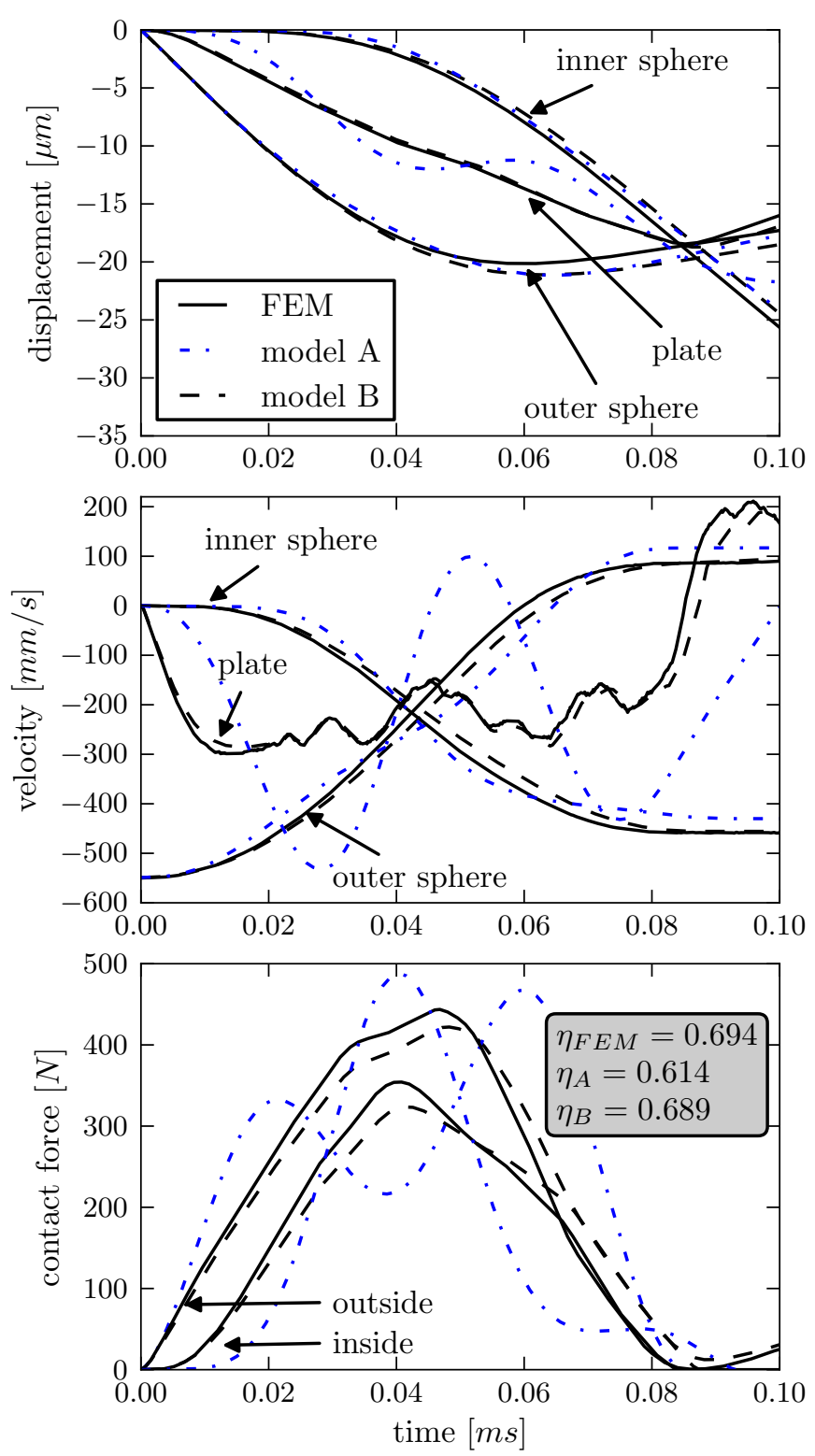

Fig. 10 Comparison between the finite element model and the multibody models with (A) 1 eigenfunction and (B) 16 eigenfunctions

left and right, aiming at the spheres. The plate which is ideally clamped in the simulation is mounted on an aluminium frame in the centre. The two spheres are suspended by thin high-modulus polyethylene wires which are connected to the spheres by small copper plates squeezed on them. The sphere which represents the actuation of the valve is released by a flap which is released by an electromagnet. For lower velocities, a different mechanism is used. A Venturi tube is used here to create a low pressure, which draws the sphere to a seal on a thin pipe. It is released by stopping the air flow through the Venturi tube. 


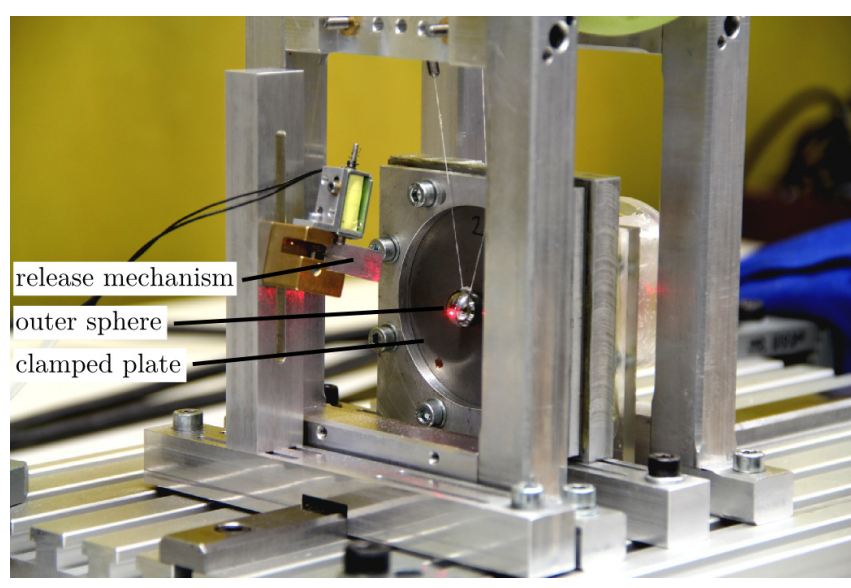

Fig. 11 Test-bed for experimental verification

The LDVs measuring the displacements and velocities are made by Polytec GmbH. One LDV is placed on the outside, measuring the sphere at the impact. This means that this sphere moves into the laser beam just before impact. The second LDV is placed on the opposite side which represents the interior of the valve, also measuring the centre of the sphere. Using both LDVs gives a displacement signal and a velocity signal for each sphere.

\subsubsection{Experimental results}

The simulated impact and the measured signals are shown in Fig. 12. The measured displacement signals are aligned in vertical direction to match the simulation results at $t=0$. We can see that it shows similar behaviour but the contact duration and the velocities after contact differ slightly.

The efficiency as defined in Eq. (1) for repeated impacts on the new and unused plates is not constant, see Fig. 13. The efficiency for sphere radii $r=10 \mathrm{~mm}$ is much lower at the first impact and increases with each additional impact until the steady-state efficiency $\bar{\eta}$ is reached. The reason for that effect are permanent changes which are caused by plastic deformations. This effect is studied in detail in Minamoto et al [9] where the same behaviour occurs. Normally, impacts with plastic deformation have a lower coefficient of restitution and therefore also a lower efficiency. So it is correct that the predicted efficiency in the finite element simulation with $69 \%$ is higher than the first data point. The steady-state efficiency $\bar{\eta}$ is typically much closer to the predicted efficiency with linear elasticity since the deformation is elastic again. Often, it is even higher because the new deformed geometry has a higher contact radius, leading to higher efficiencies as it will be presented in Section 4.2 .3

The second case with sphere radius $r=6.35 \mathrm{~mm}$ has less accurate measurements due to the smaller sphere and the different release mechanism which was used to

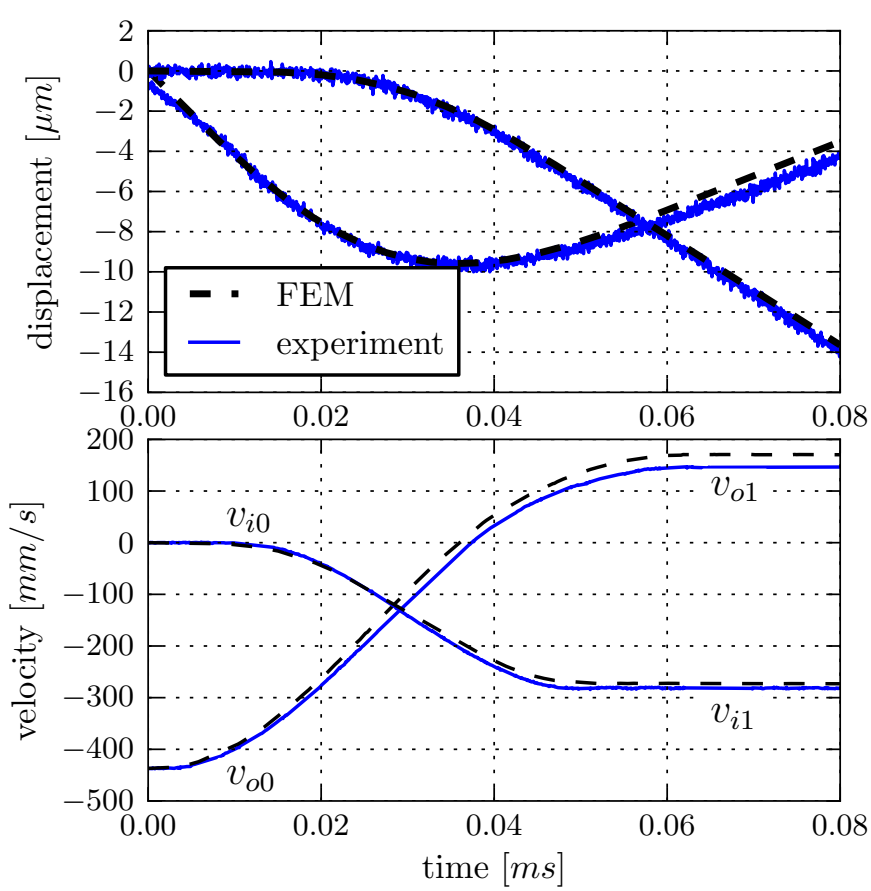

Fig. 12 Comparison of the FEM simulation and the experiment

get a lower velocity. Plastic deformations also occur here but slightly less due to the lower impact velocity. The first data points also show an increase in efficiency as expected. Here, the simulated efficiency with $39 \%$ is below the steady-state results of the experiments.

The higher variation of the points might be that the impact point is not always exactly the same, so that some of them are again influenced by plastic deformations.

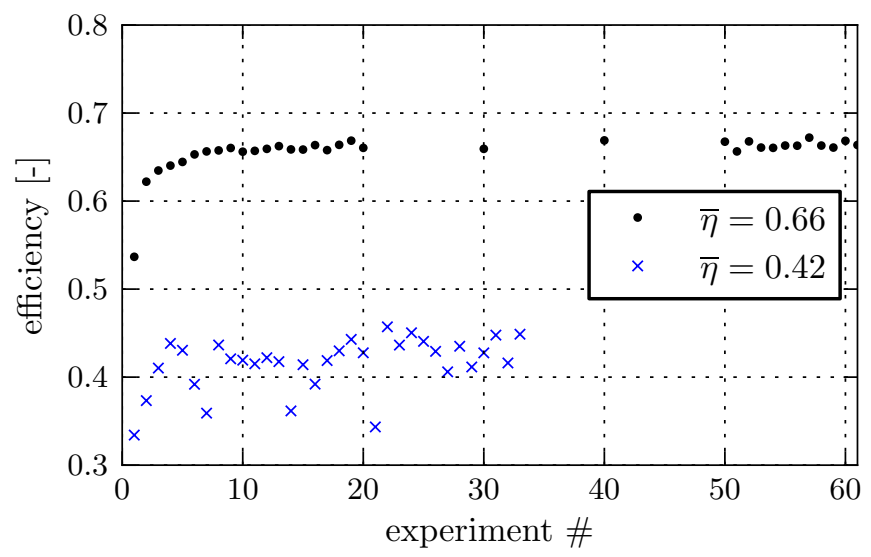

Fig. 13 Efficiency of multiple impacts for the two cases with $r=10 \mathrm{~mm}$ spheres (black dot) and $r=6.35 \mathrm{~mm}$ spheres (blue cross). 
There are several reasons why the experiments differ slightly from the simulation, although a lot of care is spent in all adjustments.

- The plate can not be clamped ideally in the experiments so that there is a vibration of the whole aluminum frame.

- The plate is not hit exactly in the centre.

- The alignment of the spheres is not exactly perpendicular to the plate, so the impact is not exactly central.

- The suspension of the spheres certainly has an influence on the movement of the spheres.

\subsection{Adding fluid flow effects to the multibody model}

In most applications of shift valves, the medium in the valve is a liquid. Therefore, the effects of a liquid in the valve must be considered in the simulation models too. The effects that are investigated are the flow resistance of moving spheres, the squeeze-film effect and the fluidadded mass.

\subsubsection{Flow resistance of spheres}

The most obvious effect of a fluid is the flow resistance of spheres in fluid flow. Here, this is inverted, during switching the sphere moves through the fluid. The typical equation for flow resistance is given by

$$
F=c_{\mathrm{W}} \rho U_{\infty}^{2} R
$$

which contains a shape-dependent factor $c_{\mathrm{W}}$, the density $\rho$ and the undisturbed velocity $U_{\infty}$. Equation $(2)$ is based on the assumption that there is only a free undisturbed flow around the considered body, which does not hold when there is a casing, where a rather complex flow takes place. Using some assumptions for material properties and shape factor, we can determine that these forces are very small compared to other effects during impact. After impact also only a slight decrease in velocity can be expected.

\subsubsection{Simulation of squeeze-film flow}

A much more important effect takes place in the gap between the sphere in the valve and the casing where the fluid is either squeezed out or sucked in. This effect is known as the squeeze-film effect which leads to high pressures in case of small gaps and high velocities. These pressures can even exceed the yield stress of steel. Therefore, a flow model for the squeeze-film flow is implemented according to the modified Reynolds equation for axisymmetric problems

$$
\frac{\partial}{\partial r}\left(\frac{r h^{3}}{\mu} \frac{\partial p}{\partial r}\right)=12 r \frac{\partial h}{\partial t} .
$$

This equation is derived for generalized Newtonian fluids in Chu et al [1. It depends on the radius $r$, pressure $p$, viscosity $\mu$ and gap $h$. The problem is axially symmetric and is reduced to one dimension by an analytic integration over the gap. This equation is solved by finite differences for each timestep. The solution gives a pressure field between the plate and the inner sphere depending on the gap height and the relative velocity. This pressure field is integrated over the surface which gives the force required for the next time step in the multibody simulation.

When the gap becomes zero, which is possible numerically, there are some parts where the bodies touch and some where there is fluid in between. Also, the pressure goes to infinity with gap height approaching zero. In cases where the bodies have an area with overlap, the geometry of the fluid domain is adjusted. When the gap becomes very small, a lot of new effects get important and the validity of Eq. (3) is limited. Furthermore, the solution with exactly zero gap is with this model almost arbitrarily dependent on the discretisation and time step size in the simulation. In reality, each surface has a roughness, so that there will most likely be some residual fluid in-between. This problem is fixed by introducing a minimal gap of $0.25 \mu \mathrm{m}$

$$
h^{*}= \begin{cases}h & \text { if } h>0.25 \mu \mathrm{m}, \\ 0.25 \mu \mathrm{m} & \text { if } 0 \leq h \leq 0.25 \mu \mathrm{m} .\end{cases}
$$

This small value is a typical roughness of high quality steel surfaces, in order to cover the smoothing effect of plastic deformations by successive impacts.

\subsubsection{Fluid-added mass effect}

It is known that elastic bodies submerged in fluids change their eigenfrequencies due to the interaction with the fluid. Our problem at hand is sensitive to eigenfrequencies; therefore, this effect must be investigated. Due to the motion of the plate, the fluid also must move which firstly increases the damping and secondly acts similar as if the mass of the plate would be higher, decreasing the frequencies. In order to capture this effect, the vibration of the plate with fluid on one side is measured with an LDV. The damping factor increases from $D=0.001$ to 0.03 and the first eigenfrequency decreases by a factor of three. The increased damping factor is simple to consider in the simulation; however, the effect on eigenfrequencies is more difficult without an accurate flow model. By premultiplying, the mass matrix of the plate elements, the first eigenfrequency can be matched according to the measurements. The influence on the higher order eigenfrequencies is less accurate with this simplification. Depending on the mode shape, the mass of the fluid that must be moved differs. However, to investigate these influences qualitatively, this model is sufficient. 


\section{Simulation results}

The simulation results concerning design decisions of the valve include different studies on parameters that influence the efficiency. Having gained an understanding for the impact process by using the described elastic multibody model, two different designs are studied in more detail. The first is based on a thin membrane and a rocker element, the second is based on a flexible seal seat.

4.1 Major effects influencing efficiency or why the multibody model works

The previously introduced elastic multibody model is detailed enough to give high accuracy, capturing the necessary effects of the impact process. We already know that the waves travel in spheres multiple times back and forth such that they behave only in a global way and can be approximated.

The behaviour of the plate is much more interesting in this respect. One might assume that a compressive wave passes through the plate and transmits the impulse completely from the outer sphere to the inner sphere. However, this can be disproved by achieving accurate results with simple plate elements without longitudinal elasticity. The reason for the influence of compressive waves which are a superposition of longitudinal eigenforms is, that in bars, the corresponding eigenfrequencies are among the first eigenfrequencies and are excited by the contact force. Plates also have longitudinal eigenfrequencies, but they are not among the first eigenfrequencies and have much higher frequencies which are less excited during impact. These high longitudinal eigenfrequencies can describe the compressive waves; however, they travel multiple times back and forth during impact, just like the waves in spheres. For a circular plate with fixed boundary condition and radius $3 \mathrm{~mm}$ the first eigenfrequency and the first longitudinal eigenfrequency depending on its thickness $t$ are given in Table 2. Basically, this shows the transition from a plate to a bar, however with fixed boundary.

Table 2 Eigenfrequencies of a fixed plate/rod with $3 \mathrm{~mm}$ radius

\begin{tabular}{rrr}
\hline $\mathrm{t}[\mathrm{mm}]$ & $1^{\text {st }} \mathrm{EF}[\mathrm{kHz}]$ & $1^{\text {st }}$ long. EF $[\mathrm{kHz}] / \#$ \\
\hline 0.1 & 29 & $1116 / 7$ \\
0.5 & 136 & $1117 / 4$ \\
3.0 & 341 & $869 / 3$ \\
6.0 & 368 & $593 / 2$ \\
12.0 & 380 & $444 / 2$ \\
24.0 & 386 & $399 / 2$ \\
\hline
\end{tabular}

By increasing the thickness of the plate the first eigenfrequency increases and the first longitudinal eigenfrequency decreases. For typical plates considered here the thickness is between 0.1 and $0.5 \mathrm{~mm}$, which means that even the first longitudinal eigenfrequency is barely excited by the impact. Therefore, we can conclude that depending on the geometry and constraints of the transmitting body for bars and plates, longitudinal eigenforms and bending eigenforms, respectively, suffice for an accurate description. Only in cases of thick plates or short bars, both must be considered.

Detailed investigations on the geometry of the plate show that the efficiency is optimal if the contact duration is approximately half the period of the first eigenfrequency. Extremely thin plates with low eigenfrequencies and low flexural rigidity have only little effect on the impact and enable high efficiencies.

\subsection{Influence of geometry and material on efficiency}

In order to better understand the impact, the influence of different parameters such as material properties, geometry and damping effects are investigated.

\subsubsection{Young's modulus}

The influence of the Young's modulus is investigated for a typical combination of parameters. The sphere radii are $2.5 \mathrm{~mm}$, the plate radius is $3 \mathrm{~mm}$ and the thickness is $0.3 \mathrm{~mm}$. The material is steel as in the scaled-up models, the Young's modulus of the three bodies is varied. In Fig. 14, the dependency for the three different bodies is shown.

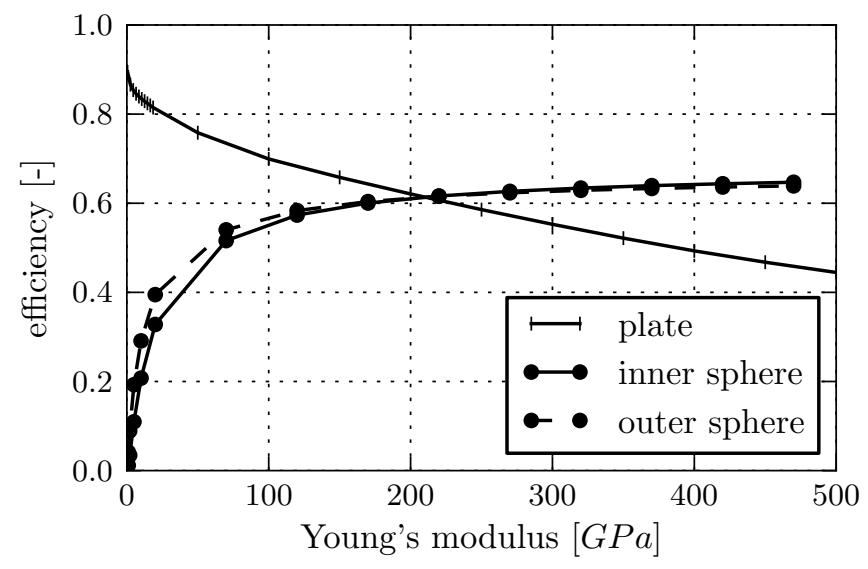

Fig. 14 Efficiency dependence on Young's modulus

The plate has a decreasing efficiency while the spheres show increasing efficiency with increasing Young's modulus. Reducing the Young's modulus for the plate reduces the flexural rigidity and therefore reduces the plates resistance in the impact process. However, the spheres show a different behaviour. An increased Young's modulus increases the contact stiffness in the Hertzian 
contact and also the contact force. This leads to a shortened contact time for the respective body. We can conclude that it is better to have a plate with a higher and spheres with a lower Young's modulus.

\subsubsection{Plate geometry}

The geometry of the plate is important for the design. A very thin plate, required for a high efficiency, is difficult to manufacture and very fragile. Therefore, a design with a thicker plate is preferred. For a typical model with a steel sphere with $1.5 \mathrm{~mm}$ radius and a titanium plate, the dependency of the efficiency on the plate thickness is plotted in Fig. 15. The efficiency decreases with increasing plate thickness for the clamped plate. The free plate also transmits energy when it is much thicker behaving more like a point mass. This is as expected from previous findings about the influence of the eigenfrequencies in Section 4.1 .

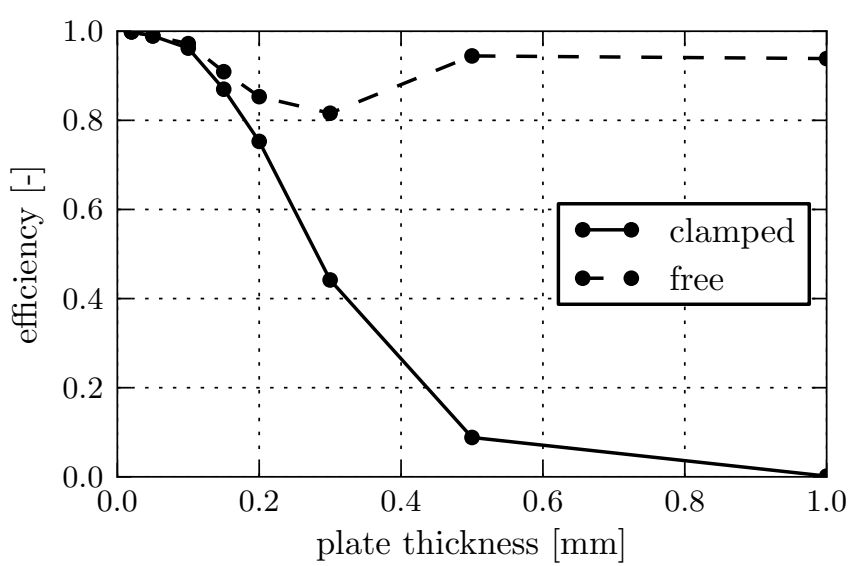

Fig. 15 Efficiency dependence on plate thickness

A variation of the plate radius for different thicknesses, as shown in Fig. 16, shows again that thinner plates have a higher efficiency. However, the thicker plates show an optimal point with highest efficiency. This is closely related to the change in the first eigenfrequency of the plate due to the changed geometry. As mentioned in Section 4.1 the first eigenfrequency of the plate must match the contact time, then the efficiency is optimal. For thinner plates, this effect is less dominant and this dependence can be neglected. If the plate must have a certain thickness with a higher flexural rigidity, the influence of the first eigenfrequency limits the plate to a minimal radius, below which the efficiency drops rapidly.

\subsubsection{Tip radius of the impacting bodies}

Not only spheres can be used as impacting elements, for example a piezo bending transducer is also possible.

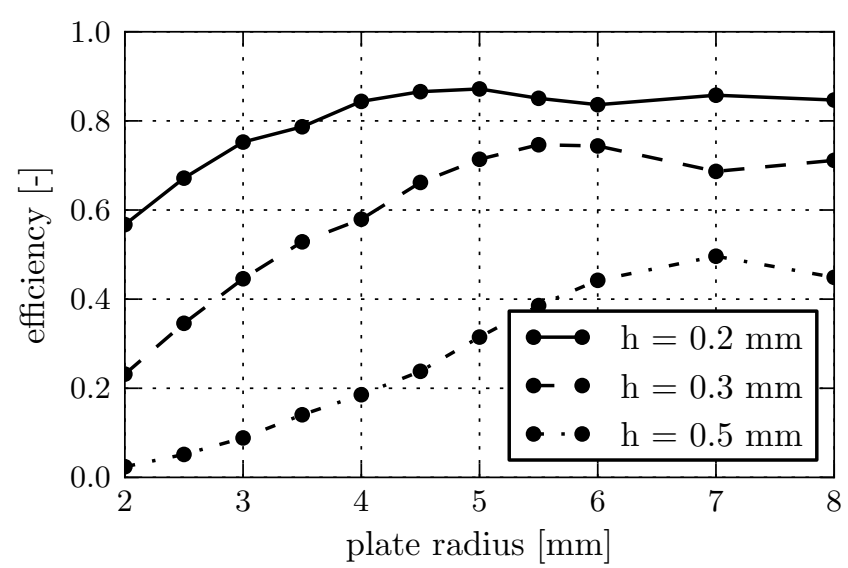

Fig. 16 Efficiency dependence on plate radius

Spheres couple the radius and the mass; however, these are two different parameters influencing the impact independently. We consider a general compact body with a mass and a tip radius with the same properties of spheres where waves cancel out. For the model used in the plate geometry investigations, with the optimal plate radius of $5.5 \mathrm{~mm}$ for a thickness of $0.3 \mathrm{~mm}$, the tip radius used in the Hertzian contact law is varied independent of the mass of the sphere. In Fig. 17, this influence is shown. The efficiency increases with increased tip radius and a larger tip radius increases the contact area. This leads typically to an increased contact force and a lower contact pressure. The higher contact force leads to a shortened contact time, which allows a higher efficiency.

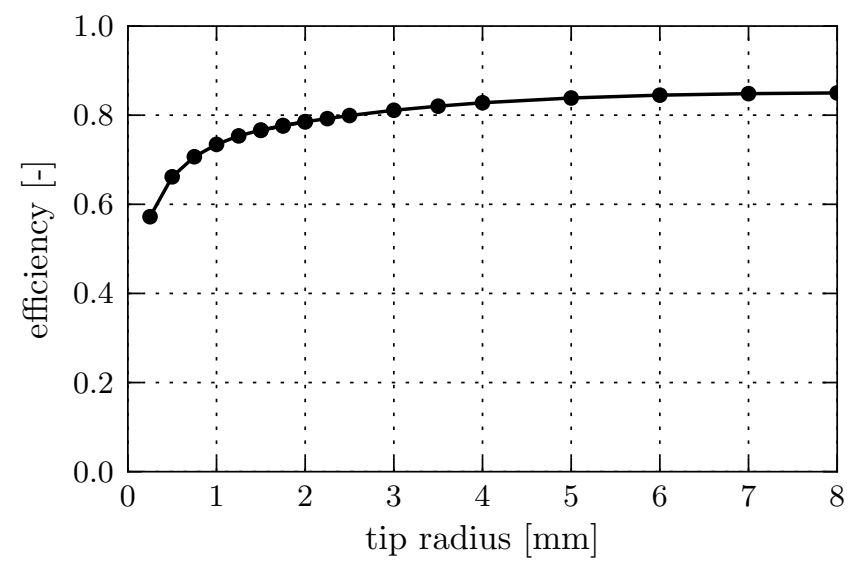

Fig. 17 Efficiency dependence on tip radius

\subsubsection{Material damping and shakedown}

Using steel or steel-like materials does not decrease the efficiency notably in respect to material damping. Polymer materials have a much higher material damping 
which leads to slightly decreased efficiency of about two per cent. This is still much less than most other effects.

For impact simulations plastic and viscoelastic material laws instead of linear elastic material laws should be used if high accuracy is required. In Minamoto et al 9 the influence of these effects is shown in detail giving very good agreement with the performed experiments. However, the influence of these complex material laws is most important for the first few impacts where plastic deformations occur. After several impacts, a stationary state is reached where no further plastic deformations occur. This effect is called shakedown. Having reached this state, the difference to the linear elastic simulation is much lower. As the focus in this paper is set on a general understanding of the impact, specifically not the first few plastic impacts, no effort is undertaken to get detailed material data for such simulations.

\subsubsection{Influence of fluidic effects on efficiency}

Fluids always reduce the efficiency either by increased damping, the squeeze film effect which also has a dampening effect and last but not least reduced eigenfrequencies of the plate. The increased damping reduces the efficiency only by up to $3 \%$. But the changed eigenfrequencies can have a much larger influence. This depends on the sensitivity of the model on the first eigenfrequency. Models with high flexural rigidity typically are more sensitive, the influence can be up to about $30 \%$ here. The fluid models are only accurate enough to give qualitative results so that it does not make sense to optimise the valve to increase the efficiency only slightly. It can be said, that a design with a reasonable 'dry' efficiency should also work with different kinds of fluids, because the efficiency drop is typically not very high. For more detailed answers also more detailed models for the fluids are required.

\subsection{Rocker element model}

The previous investigations about different influencing parameters lead to a first design with maximised efficiency as described in Section 2.3.1. However, not just the geometry and materials are chosen in a way that the efficiency is high, but also the actuator is a piezo bending transducer now. The plate is in this case not so much a plate, as thin titanium alloy membrane with a thickness of $0.1 \mathrm{~mm}$ and a radius of approximately $3.1 \mathrm{~mm}$. The inner sphere is made of sapphire and has a radius of $2.0 \mathrm{~mm}$. The material parameters are listed in Table 3 .

Simulating this model requires that the piezo bending transducer is modelled. For that, an approximate model using beam elements and a point mass is used. The first eigenfrequency of the beam with approximately $500 \mathrm{~Hz}$ and its stiffness of $0.2 \mathrm{~mm}$ deflection at $0.5 \mathrm{~N}$
Table 3 Material properties for the materials used in the rocker element model

\begin{tabular}{l|rr} 
& Titanium alloy & Sapphire \\
\hline Young's mod. $\left[\mathrm{N} / \mathrm{mm}^{2}\right]$ & 115000 & 350000 \\
density $\left[\mathrm{kg} / \mathrm{m}^{3}\right]$ & 4430 & 3980 \\
Poisson's ratio $[-]$ & 0.37 & 0.27 \\
\hline
\end{tabular}

was measured. The beam model is adapted to behave in the same way. The beam is deflected by piezo bending transducers and released just before impact. For a velocity of $550 \mathrm{~mm} / \mathrm{s}$ at the impact, a torque of $4 \mathrm{Nmm}$ is required. The point mass at the tip of the bending transducer is $128 \mathrm{mg}$, which is approximately the same as the mass of the inner sphere. The tip radius of the point mass on the bending transducer is $4 \mathrm{~mm}$ which is rather high and gives a slightly higher efficiency. For comparison, a sphere exactly the same as the inner sphere, is used as actuation.

Both models are set up such that the inner sphere has a velocity of approximately $555 \mathrm{~mm} / \mathrm{s}$ after impact. The simulation with two spheres gives the highest possible efficiency of $97.5 \%$. Using the bending transducer as actuator, it is difficult to define an efficiency. Taking the kinetic energy of the whole bending transducer gives an efficiency of $66 \%$ and using the strain energy just before the release gives $41 \%$. This shows that this design should work, even with the decreased efficiency with the piezo bending transducer as actuator which requires a higher energy to achieve the same result.

\subsection{Contact simulation of a design with a flexible mounted seal seat}

Based on the previous findings, a new design based on a flexible mounted seal seat, as introduced in Section 2.3.2, is simulated. Due to the strong influence of the more complex geometry, a 3D finite element model is necessary. In the previous investigations of major effects influencing efficiency, we have seen that flexible plate elements with a low Young's modulus are advantageous for the efficiency. After iterating through several designs based on a flexible support, finally the following design was created. The sphere and the actuator at the bottom are made of steel. The whole seal seat is made of polymer. In the simulation the impact is initiated by a given initial velocity for the impacting body. After the impact, the velocity of the sphere is investigated. The neglected spring would force the sphere back down and ideally into the position on the right. In this short timescale simulations, only the vector of the velocity after impact is investigated. In Fig. 18 the velocity direction is displayed by a red arrow which shows that the sphere moves in the wrong direction.

This is due to the resulting moment on the seal seat by the contact forces, which have a horizontal offset. This moment rotates the seal seat which changes the di- 


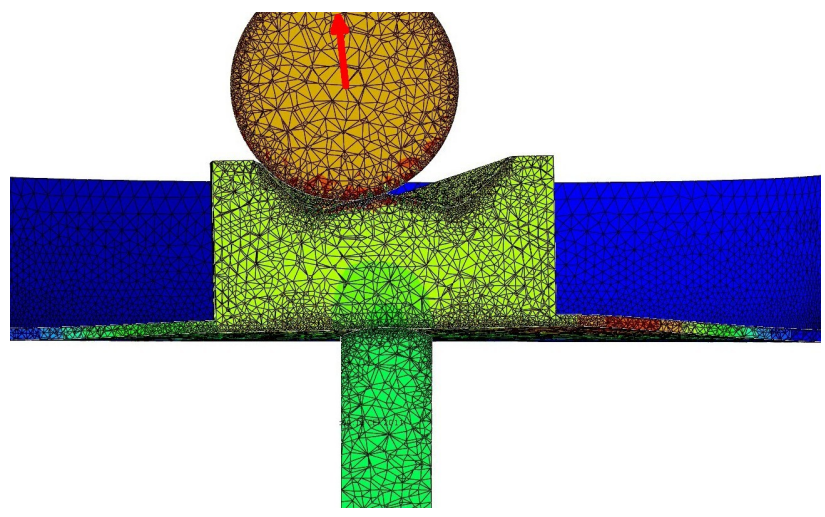

Fig. 18 Simulation result for the flexible mounted seal seat

rection of the movement. Also the contact forces are not equally distributed at the bottom of the sphere. This also leads to a resulting force straight up and slightly to the left instead of the intended direction given by the shape of the seal seat. Measurements with a high speed camera are discussed later in Section 5.2. Several changes to the design in Fig. 19 lead to an improved behaviour. Firstly, the design is inverted and weakened at the centre such that the centre part gets bended and therefore changes direction of the resulting contact force. Secondly, the impacting body does not hit at the centre but on both sides of the centre part. This prevents the seal seat from rotating in the wrong direction. The results of this optimised design lead to a velocity vector approximately in the intended direction.

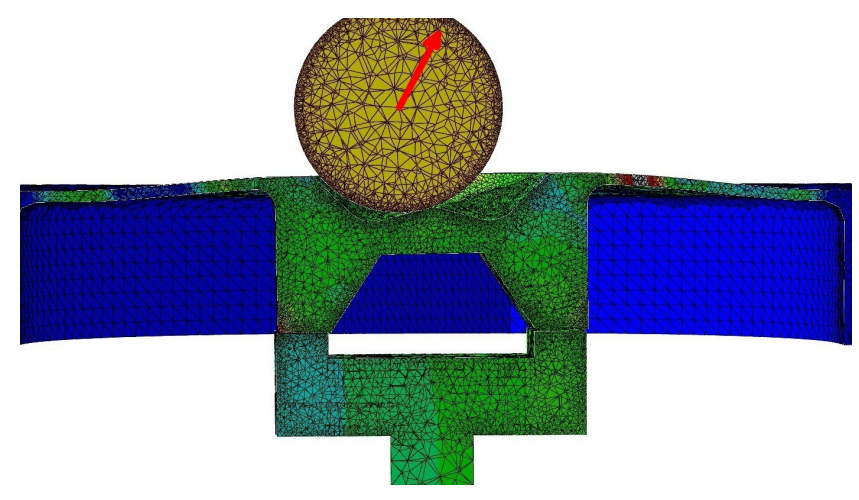

Fig. 19 Simulation result for the improved design

\section{Experiments with the prototypes}

For both valve designs that are presented in Section 2 demonstrators were set up. The valve demonstrator with the rocker element thereby could be set up either with piezo stack actuators or with piezo bending actuators as can be seen in Figs. 20 and 21

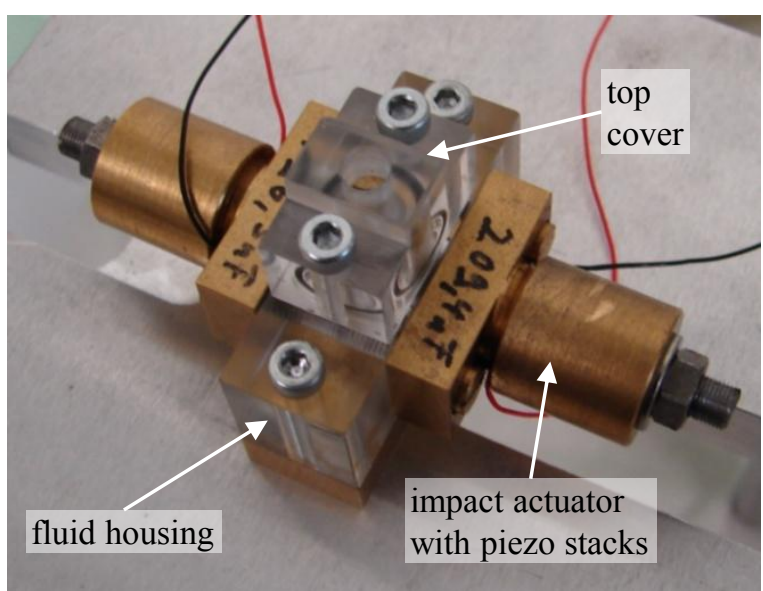

Fig. 20 Valve demonstrator with a rocker element and piezo stack actuators

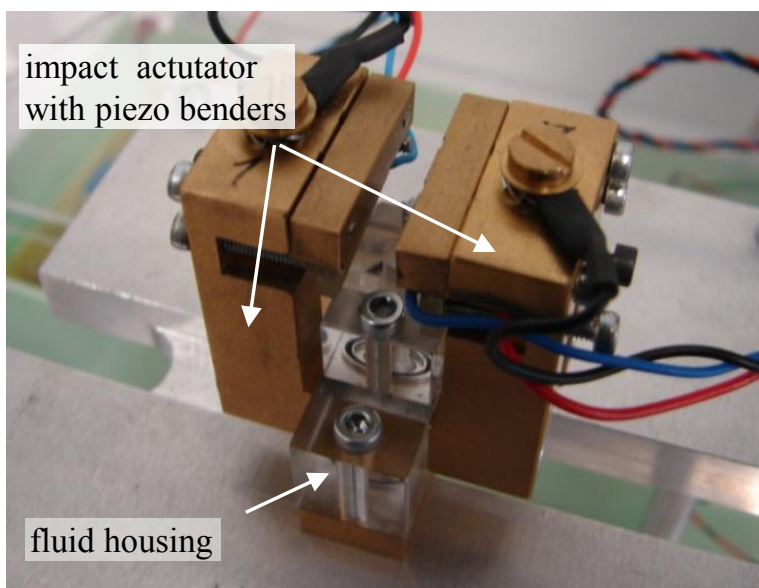

Fig. 21 Valve demonstrator with a rocker element and piezo bending actuators

The valve demonstrator with the flexible mounted seal seat is shown in Fig. 22 .

The electronic supply for the demonstrators also was set up, whereby the main components of the circuit were already chosen as miniaturized parts to verify the miniaturization potential of the whole concept.

5.1 Experiments with the valve demonstrator with a rocker element

Several experiments were carried out with the demonstrator with a rocker element, starting with the most important tests about the shift function, long-term tests and a test about the robustness against vibrations.

\subsubsection{Verification of the shift function}

The shift function of the valve was verified for an orientation of the valve body according to the three coordinate directions. Generally, the shift function was 


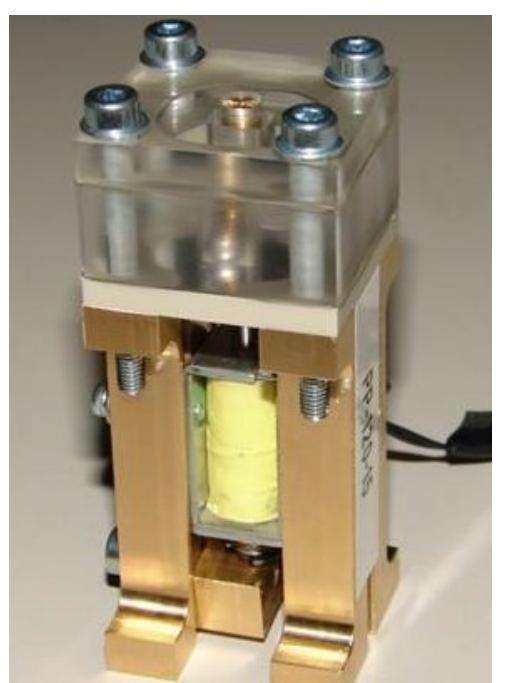

Fig. 22 Valve demonstrator with a flexible mounted seal seat

verified also in the worst-case situation, with gravity accelerating the sphere down. Sometimes, it was observed that the sphere fell back to the lower position when the impact applied by the lower actuator was too low to overcome the gravitational forces of the sphere and the compression forces of the spring. By changing the pre-tension of the spring, this effect could be eliminated.

\subsubsection{Long-term test}

The next step was a long-term test that was performed with the valve demonstrator in order to show the reliable shifting behaviour of the system. Therefore, the transmission of the spring force to the sphere had to be optimized. As can be seen in Fig. 4, the last turn of the spring covers the upper part of the sphere, resulting in a quite large contact area, where friction between the sphere and the spring occurs. In combination with the friction between the sphere and the rocker, this can cause an unintended stopping of the sphere during the shift process in a middle position which of course means a total failure of the valve. In order to overcome this problem, a point transmission of the force to the sphere was realized by a small plunger in a blind hole in the sphere as shown in Fig. 23.

With this set-up it was possible to reach over 2 million shifting operations in a long-term test, performed with piezo bending impact actuators. During this longterm test, it was observed only about 8 times that the sphere stopped in a middle position, but it could be moved to the end position again by a repeated actuation of one impact actuator at a high frequency, and therefore, no total failure of the valve occurred.

Another question that was investigated was the wear of the components of the impact actuator. As can be seen in Figs. 24 and 25, no significant wear can be ob-

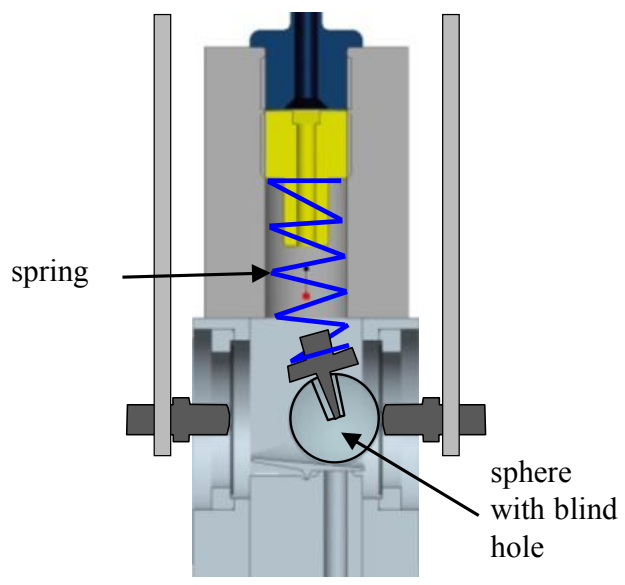

Fig. 23 Point transmission of the spring force to the sphere by a plunger

served. Only the tip of the plunger that was mounted on the piezo bending actuator shows a small flattening and a marking on the membrane that transmits the impact energy is visible.
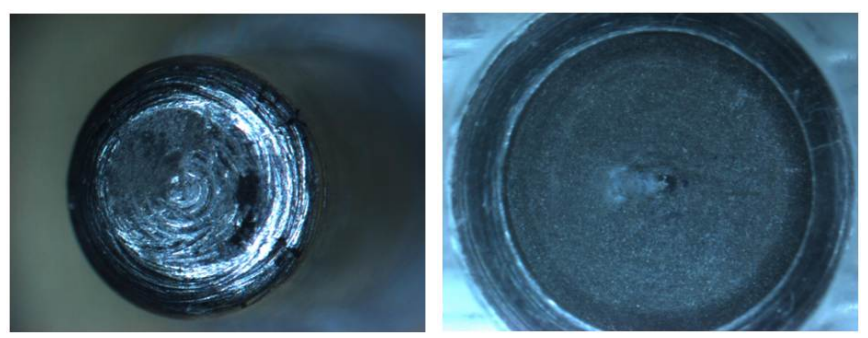

Fig. 24 Small flattening on Fig. 25 Marking on the memthe tip of the plunger of the brane after 2 million shifting impact actuator after 2 million operations shifting operations

\subsubsection{Robustness against vibrations}

The last test that was performed was a vibration test on a shaker in order to investigate the robustness of the setup against vibrations. This is interesting because the valve should not shift unintended when an acceleration is applied to the valve body, e.g. by external vibrations or shocks. However, it was observed that the robustness against external vibrations is not yet very high in this set-up. The rocker element can be moved out of its position in the valve chamber by external accelerations that are in the range of 10 times the gravitational constant. A solution of this problem has to be found probably by changing the design of the inner walls of the valve chamber so that the free moving space of the rocker is constricted. 


\subsection{High-speed camera measurements}

The shift valve with flexible mounted seal seat was manufactured and high-speed camera recordings were made. The recordings were made with frame rates between 2000 and 4000 frames per second. In Fig. 26 is an image series, representing the motion of the sphere after impact of the air-filled shift valve demonstrator. The images were always made at points where the motion changes direction. In contrast to the expected motion, the impact accelerates the sphere mainly in upper vertical direction as predicted in Section 4.4

The sphere is decelerated by the spring and pushed back down where it hits an edge at the bottom which enables a horizontal movement to the end position. This is obviously a very sensitive design. These records were done without fluid filling.
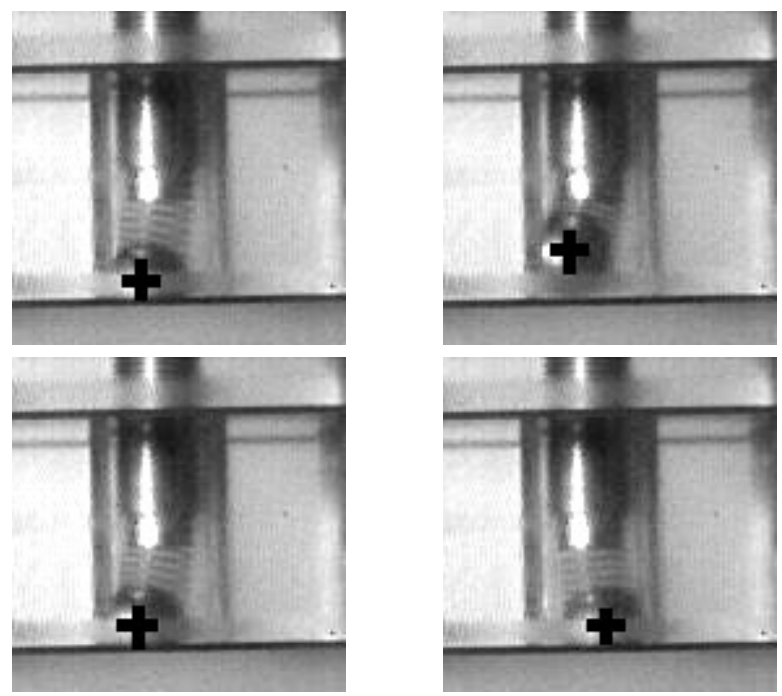

Fig. 26 Motion of the sphere in the valve (reversal points)

When the flexible seal seat is not hit central, the valve switches also with fluid inside. However, the goal is to find a design with just one actuator; therefore, only the improved design from Section 4.4 might give a satisfactory result.

\section{Conclusions}

The design of a miniaturised shift valve with impact actuation, supported by numerical simulation of elastic multibody models and finite element models, as well as experiments and tests, were shown. An elastic multibody model was created to model the impact where the relevant effects were easier to investigate in terms of modelling and computational time and because of the simplifications typical for such models. For example, effects that are not modelled in the elastic multi- body model, cannot be significant. Otherwise, the elastic multibody model would not give accurate results. One of these effects is the plate elasticity in longitudinal direction which is neglected by using plate elements to model the plate in the elastic multibody model. The elastic multibody model was extended by different effects such as support for squeeze-film flow and fluidadded mass effects. Also, an approximate model for the dynamics of a piezo bending transducer was added. Experiments to validate the simulation models were performed showing general agreement. For better results, detailed material data is required to model the material with nonlinear material behaviour. With the gained knowledge, different designs for the shift valve were developed and tested. Experiments with laser-dopplervibrometers and a high speed camera could show that the predicted behaviour of the shift valve is correct.

\section{Acknowledgements}

The IGF $341 \mathrm{ZN}$ research project is funded within the program of promoting "pre-competitive joint research (IGF)" by the German Federal Ministry of Economics and Technology BMWi based on a decision of the German Bundestag. It was carried out under the umbrella of the German Federation of Industrial Co-operative Research Associations (AiF). This support is highly appreciated. We also want to thank Dipl.-Ing. A. Bülau for the valuable contribution to our research by designing and characterizing the shift valve with the flexible mounted seal seat.

\section{References}

1. Chu HM, Chen JL, Hsu HC, Li WL (2007) Elastohydrodynamic lubrication of circular contacts at impact loading with generalized Newtonian lubricants. Tribology Letters 29:1-9

2. Eberhard P, Fischer C (2011) Simulation of a micro shift valve with impact actuation. In: Samin JC, Fisette P (eds) Proceedings of the ECCOMAS Thematic Conference on Multibody Dynamics 2011, Brussels, Belgium

3. Fehr J, Eberhard P (2010) Error-controlled model reduction in flexible multibody dynamics. Journal of Computational and Nonlinear Dynamics 5(3):031,005-1- 031,005-8

4. Fritz KP (2012) Untersuchungen zu miniaturisierten Schaltventilen mit Medientrennung und Stoßantrieb (in German). Dissertation IZFM der Universität Stuttgart, Reihe: Ingenieurwissenschaften, Verlag Dr. Hut, München

5. Fritz KP, Fischer C (2012) Miniaturisiertes Schaltventil mit Medientrennung (in German). AiF final report

6. Goldsmith W (1960) Impact: the theory and physical behaviour of colliding solids. Edward Arnold, London

7. Hinton E (1975) The dynamic transient analysis of axisymmetric circular plates by the finite element method. Journal of Sound and Vibration 46:465-472

8. Jones E, Oliphant T, Peterson P, et al (2011) SciPy: Open source scientific tools for Python. URL http://www . scipy . org/ 
9. Minamoto H, Seifried R, Eberhard P, Kawamura S (2011) Analysis of repeated impacts on a steel rod with viscoplastic material behavior. European Journal of Mechanics A/Solids 30(3):336-344

10. Seifried R (2005) Numerische und Experimentelle Stoßanalyse für Mehrkörpersysteme (in German). Dissertation, Schriften aus dem Institut für Technische und Numerische Mechanik der Universität Stuttgart, Band 2, Shaker Verlag, Aachen

11. Seifried R, Schiehlen W, Eberhard P (2010) The role of the coefficient of restitution on impact problems in multibody dynamics. Proceedings of the Institution of Mechanical Engineers, Part K: Journal of Multi-body Dynamics 224(3):279-306, DOI 10.1243/14644193JMBD239

12. SymPy Development Team (2011) SymPy: Python library for symbolic mathematics. URL http://www. sympy.org

13. Szabó I (1960) Höhere Technische Mechanik (in German), 3rd edn. Edward Arnold, London

14. Zienkiewicz O, Bauer J, Morgan K, Onate E (1977) A simple and efficient element for axisymmetric shells. International Journal for Numerical Methods in Engineering 11:1545-1558 